\title{
RECURSIVE DYNAMIC PROGRAMMING: HEURISTIC RULES, BOUNDING AND STATE SPACE REDUCTION
}

\author{
Henrik Kure \\ Dina, Danish Informatics Network In the Agricultural Sciences \\ Royal Veterinary and Agricultural University \\ Bulowsvej 13, DK-1870 Frb., Copenhagen.E-mail: kure@dina.kvl.dk
}

\begin{abstract}
An alternative approach towards dynamic programming (DP) is presented: Recursions. A basic deterministic model and solution function are defined and the model is generalized to include stochastic processes, with the traditional stochastic DP model as a special case. Heuristic rules are included in the models simply as restrictions on decision spaces and a small slaughter pig marketing planning example illustrates the potential state space reductions induced by such rules.
\end{abstract}

Keywords: Dynamic programming, recursive algorithms, heuristics, stochastic modelling.

\section{INTRODUCTION}

Dynamic Programming (DP) is a general optimization principle, formulated by Bellman (1957). The principle is usually related to the field of Operations Research (OR), but the underlying and rather intuitive "Principle of optimality" (Bellman, 1957) is essential to several specific optimization techniques, including $\mathrm{AI}$ algorithms as $A^{*}$ (Winston, 1992) and the solution methods of Influence Diagrams and Valuation Networks (Shenoy, 1992).

Value Iteration (VI) is (at least in OR) the best known and widely used DP technique. In this paper an alternative and more general approach towards DP is presented: Recursions. The approach (Recursive Dynamic Programming, RDP) is basically a direct implementation of the fundamental functional equation of DP (as defined by eg. Nemhauser (1966)):

$$
f_{n}\left(x_{n}\right)=\min _{d_{n} \in D_{n}}\left[r_{n}\left(x_{n}, d_{n}\right)+f_{n-1}\left(t_{n}\left(x_{n}, d_{n}\right)\right)\right]
$$

where $n$ is the stage number,

$x_{n}$ is the state ( $n=0$ indicates a goal state),

$f_{n}$ is the solution function at stage $n$. Returns the solution to the problem associated with $x_{n}\left(f_{0}\right.$ $\left(x_{0}\right)$ is per definition a known value), $d_{n}$ is a decision, chosen from the set of valid decisions, $D_{n}$,

$r_{n}$ is the gain function and

$t_{n}$ is the transition function.

It must be emphasized that this approach neither is nor intend to be novel, but it appears to be relatively unknown to OR and agricultural scientists (Kure, 1995). The purpose of this paper is to show the simplicity and the potential computational benefits of RDP.

The approach has, as will be shown, several theoretical and applicational implications. The methods presented may be applied to virtual any problem that might be solved using VI, including agricultural planing/optimization problems like facility management, replacement problems and farm operations scheduling. The methods (as defined in this paper) have been applied to simple illustrative problems and a slaughter pig marketing decision support system which is under construction.

All models and functions in this paper are specified and defined using the syntax of the specification language, VDM (see eg. Jones (1990) for an introduction) which in many aspects is equivalent to functional languages as SML. 


\section{RECURSIVE DYNAMIC PROGRAMMING}

An example might illustrate the model, the problem, the method and some implications of RDP:

Consider a road map represented as a directed graph as shown in fig. 1. Each node (or state) in the graph represents a crossroads and each (directed) edge represents a piece of one-way road. A decision prescribes what edge to follow from a given node. Node $\mathrm{A}$ is defined as the start node. Node B has no successors; it is a goal node. Edges might be interpreted as a pair of functions:

a) A transformation function:

$t$ : State $\times$ Decision $\rightarrow$ State

returning a new node/state and

b) a gain function:

$g:$ State $\times$ Decision $\rightarrow$ Gain

returning a gain (ie. the length of an edge).

A policy is (in this presentation) defined as an assignment of a (valid) decision to every node in a sub-set of nodes in the graph and a complete policy assigns a decision to every node (except goal nodes) in the graph. The optimization problem to be considered here is: What is the shortest path from $A$ to $B$ ?

\subsection{The Data Model}

Based on these basic components a deterministic data model/data type of a system is formulated as a finite mapping from states into a finite mapping from decisions into gains and (succeeding) states:

$$
\begin{aligned}
& \text { DPModel }=\text { State } \stackrel{\text { m }}{\rightarrow} \text { Decision } \stackrel{\text { m }}{\rightarrow} \text { Gain } \times \text { State } \\
& \text { inv }(d p m) \ldots
\end{aligned}
$$

where the invariant must restrict the model to be finite and cycle free (see Kure (1995) for a definition). The model will later be modified to be based on functions instead of finite mappings. In addition to the data model of the system a representation of a policy is required:

$$
\text { Policy }=\text { State } \stackrel{\mathrm{m}}{\rightarrow} \text { Decision }
$$

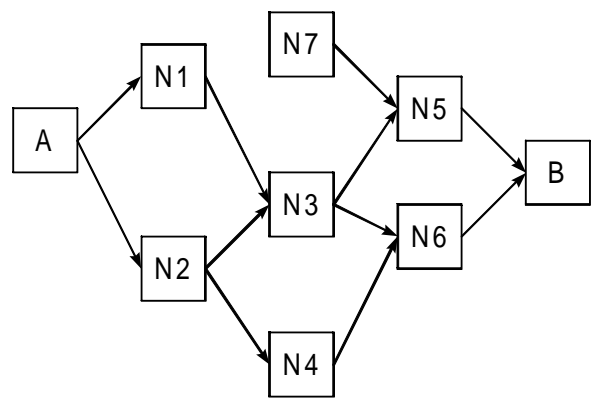

Fig. 1. A road map.

\subsection{The Problem}

In order to formulate and solve the problem, a set of basic functions is specified (see Jones (1990)):

$$
\begin{aligned}
& \text { complete_pol (dpm: DPModel, p: Policy) r: boolean } \\
& \text { post } \quad r=(\operatorname{dom} p=\operatorname{dom} d p m \wedge \\
& \forall s t \in \operatorname{dom} p \cdot p(s t) \in \operatorname{dom} d p m(s t)) \\
& \text { all_pol (dpm: DPModel) r: Policy-set } \\
& \text { post } \quad r=\{p \in \text { Policy } \mid \text { complete_pol }(d p m, p)\} \\
& \text { combine: Gain } \times \text { Gain } \rightarrow \text { Gain }
\end{aligned}
$$

all_pol returns all complete policies applicable to a given instance, $d p m$, of the model. The function comb is not yet defined (in the example it is defined as a simple addition of the arguments), but is used in the definition (see Jones (1990) for an introduction to direct definitions in VDM) of the objective function:

$$
\begin{aligned}
& \text { objfct: } \text { DPModel } \times \text { Policy } \times \text { State } \rightarrow \text { Gain } \\
& \text { objfct }(d p m, p, s t) \triangleq \\
& \text { let }\left(g, n \_s t\right)=d p m(s t)(p(s t)) \\
& \text { in } \quad \text { if } \quad n \_s t \notin \operatorname{dom} p \text { then } g \\
& \quad \text { else comb }\left(g, o b j f c t\left(d p m, p, n \_s t\right)\right)
\end{aligned}
$$

Given a instance of the model (dpm: DPModel) and a state (st: State), the problem is to minimize the objective function objfct with respect to all complete policies $\left(p \in a l l \_p o l(d p m)\right)$ :

$$
\min \left\{\operatorname{objfct}(d p m, p, s t) \mid p \in a l l \_p o l(d p m)\right\}
$$

In other words the goal is to find a solution function, solution, that satisfies the specification:

$$
\begin{aligned}
& \text { solution (dpm: DPModel, st: State) } r \text { : Gain } \\
& \text { pre } \quad \text { st } \in \text { dom } d p m \\
& \text { post } \quad r=\min \{\text { objfct }(d p m, p, s t) \mid p \in \text { all_pol }(d p m)\}
\end{aligned}
$$

The state space is defined as the total set of states (except goal states) in the model and a decision space as the total set of valid decisions in a given state:

$$
\begin{aligned}
& \text { state_space }(d p m) \triangleq \operatorname{dom} d p m \\
& \text { decision_space }(d p m, s t) \triangleq \operatorname{dom} d p m(s t)
\end{aligned}
$$

\subsection{A solution function}

By introducing two new functions a solution function may be derived (see Kure (1995) where a proof based on a mathematically interpretation of Bellman's Principle of Optimality is given):

$$
\begin{aligned}
& \text { min_two: } Y \times Y \rightarrow Y \\
& \text { min_two }(a, b) \triangleq \text { if } a<b \text { then } a \text { else } b
\end{aligned}
$$




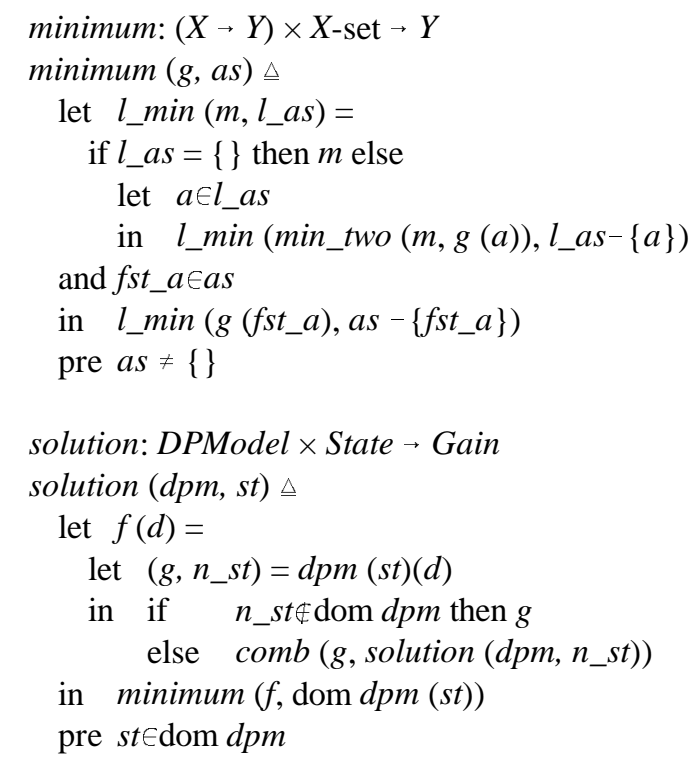

The function minimum minimizes the returns of the function $g$ over as, a finite set of arguments to $g$. In solution minimum is utilized to minimize $f$ over the finite set of valid decisions, dom $d p m$ ( $s t$, where $f$ returns the optimal gain of executing a decision, $d$. The problem of finding the shortest path from A to B in fig. 1 is solved recursively starting in the start state (A).

\subsection{Memory functions}

As shown in the example, RDP consists of a recursively decomposition of a problem into and solving of subproblems, but the solution function, solution, does not prevent the same problem to be solved more than once (ie. in the example different paths may lead to the same node). This (possibly inefficient) resolving of the same problem may be eliminated by keeping a record of known solutions: If a solution is recorded, it is already known and may be used directly. Otherwise the problem must be solved and the result saved in the record for later use. This use of record keeping or use of a memory function (Brassard and Bratley, 1988) is an (or the) essential part of DP and may result in great savings in computing time.

The record of solutions might be represented as a map:

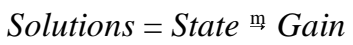

If knowledge of the policy leading to the solution is required, this optimal policy might be represented as an extension to the record of solutions:

$$
\text { SolutionsOptPolicy }=\text { State } \stackrel{\text { m }}{\rightarrow} \text { Gain } \times \text { Decision }
$$

All solution functions in this paper might be extended with memory functions (see Kure (1995) for direct definitions).

\subsection{Function based model}

The basic data model may be modified to be based on functions instead of (finite) maps:

$$
\begin{aligned}
& \text { NotInDomain }=\text { State } \rightarrow \text { boolean } \\
& \text { DecisionSpace }=\text { State } \rightarrow \text { Decision }- \text { set } \\
& \text { Consq }=\text { State } \times \text { Decision } \rightarrow \text { State } \times \text { Gain } \\
& \text { DPModelFunc }= \\
& \quad \text { NotInDomain } \times \text { DecisionSpace } \times \text { Consq } \\
& \text { inv }(\text { dpm }) \triangle \ldots
\end{aligned}
$$

where NotInDomain restricts the domain of the model (or the problem space), DecisionSpace returns the decision space of a given state and Consq returns the consequences (ie. gain and succeeding state) of executing a decision.

Based on this data model the basic solution function may be changed into:

$$
\begin{aligned}
& \text { solution_func: DPModelFunc } \times \text { State } \rightarrow \text { Gain } \\
& \text { solution_func }(\text { dpm, st }) \triangleq \\
& \text { let } \quad(\text { not_in_dom, decs, consq })=d p m \\
& \text { and } f(d)= \\
& \text { let }\left(g, n \_s t\right)=\text { consq }(s t, d) \\
& \text { in if not_in_dom }\left(n \_s t\right) \text { then } g \\
& \quad \text { else comb }\left(g, \text { solution_func }\left(d p m, n \_s t\right)\right) \\
& \text { in minimum }(f, \text { decs }(s t))
\end{aligned}
$$

The flexibility of this model and solution function will be exemplified in section 4.

Selected parts of a SML-implementation (see Paulson (1991) for an introduction) is shown in the appendix. The function not in dom (of type: NotInDomain) is in this implementation a global function and not a part of the data model.

\section{GENERALIZED RDP}

The basic deterministic model, DPModel is easily generalized to include stochastic processes.

A general summation function used in the definitions is defined as:

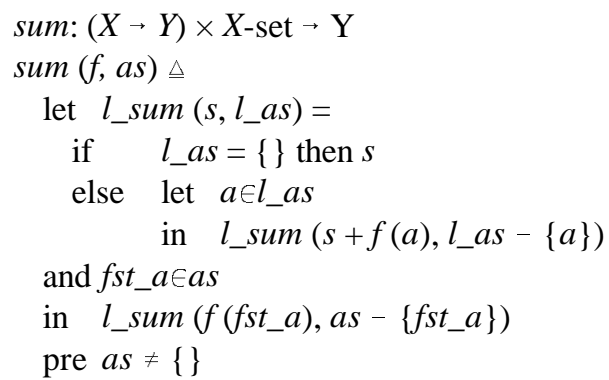




\subsection{The model}

Let random events (or states) be represented by the data type ChanceState. The general model might then be defined as:

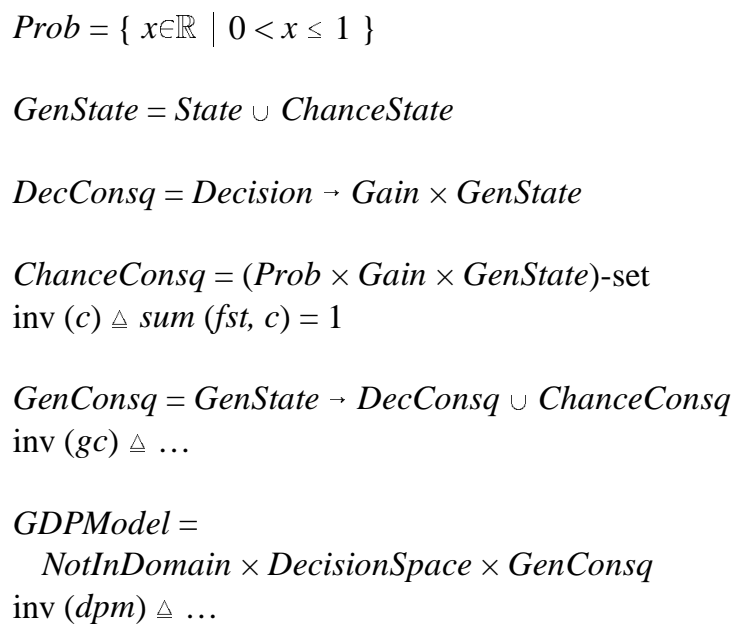

The graph in fig. 2 is an example of an instance of this model and it illustrates an important feature of the model: Decision nodes and chance nodes might appear in any order. A stochastic DP model (see eg. Nemhauser, 1966) is seen to be a special case of GDPModel, in which a State always (unless it is a goal state) is succeeded by ChanceStates and vice versa. In this special case the representation of chance states is superfluous and the model might be reformulated as:
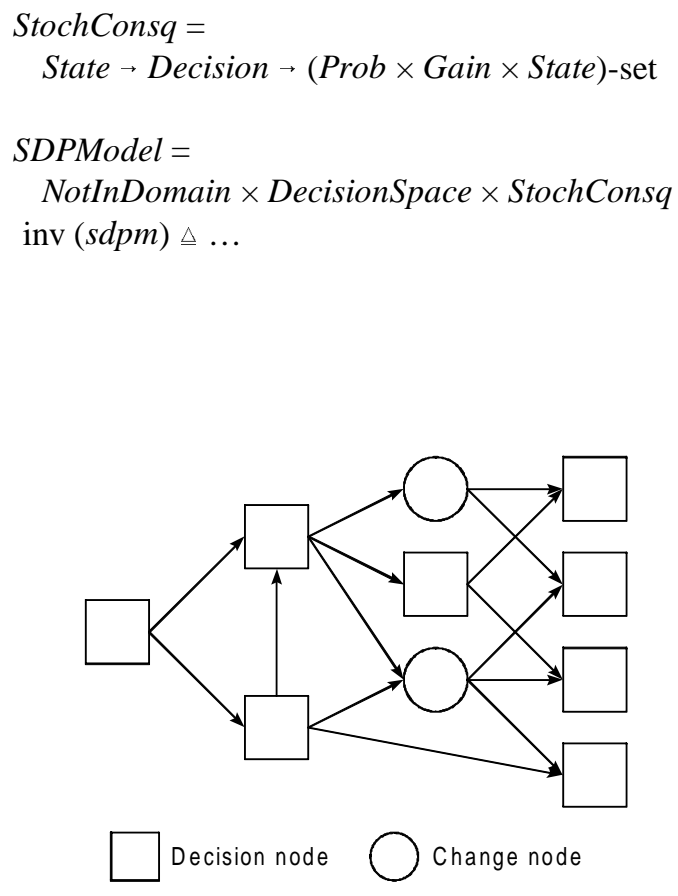

Fig. 2. An instance of a General DP model. Nodes represent values (and not variables as in Bayesian Networks).

\subsection{The solution function}

A solution function for the general model is defined as:

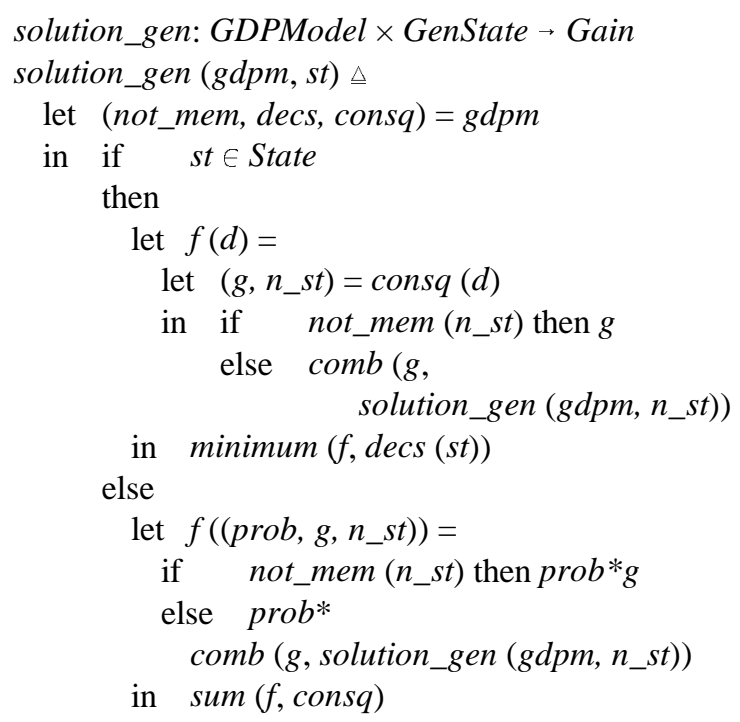

which is seen to be an extended version of solution_func: if the state st is a ChanceState the weighted gains are summed up; if not, the gains are (exactly as in solution_func) minimized.

\section{HEURISTIC RULES AND BOUNDING}

Dynamic programming is in itself a very powerful technique, but in some situations heuristic reasoning might (or even has to) be applied in order to reduce state spaces (and to make incomputable problems computable). The solution functions presented in section 2 and 3 might be extended to include heuristic reasoning.

\subsection{Heuristic rules}

Without significantly effecting the optimal solution a heuristic rule for each state in the state space reduces the decision space and consequently the state space itself. These rules (and restrictions on decision spaces in general) are simply included in the models as modifications of DecisionSpace.

A road map (continued). The example might illustrate how restrictions on decision spaces might reduce the state space. The rule/restriction applied to the example is as follows: "Always follow the road at the left if you have the option." Fig. 3 illustrates this situation; roads "at the right" have been erased from the original graph (fig. 1) and only one path from A to B remains. The state space has been reduced to 4 states (A, N1, N3 and N5) and the recursive solution function is "reduced" to a simple summation function; $m i n \_t w o$ is never called. 


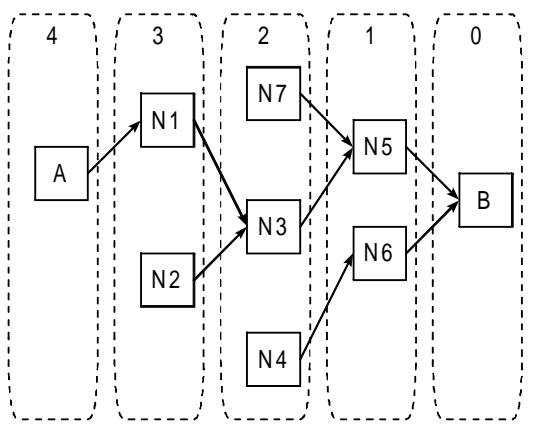

Fig. 3. The graph of fig. 1 partitioned into stages and applied the rule: "always follow the road at the left if you have the option."

Slaughter pig marketing. Another example might illustrate the potential magnitude of the state space reductions: A farmer has a barn which is partitioned into 3 sections; each section containing from 0 to 5 pigs (of same age). Sections has to be emptied after no more than 15 weeks (this is actually a decision space restriction) and sections are assumed equal. Pigs might be marketed at any time. The problem is: when is it time to market individual pigs and when is it time to empty a section and insert a new group of 5 piglets? The size of the state space of a section is $15^{*} 6=90$ and the size of the state space of the barn pr. stage is: $90 * 89 * 88+90 * 1 * 89+$ $90 * 1 * 1=\underline{712,980}$. A set of restrictions /heuristic rules might describe the fact that many of these states (like eg. "no pigs in any section") under normal conditions are very rare:

- Max 1 insertion pr. week (labor restrictions).

- No deliveries before week 13 (from experience/ growth models).

- One pig is delivered pr. section pr. week from the age of 13 weeks (from experience).

Given these restrictions the total number of states pr. stage is 1,690 , but after optimization (40 weeks) the actual maximum number of states per stage is $\underline{462}$.

Value Iteration. In VI or backward multistage problem solving (Nemhauser, 1966) the problem space is partitioned into stages. Each stage contains problems that might be solved independently and in any order. The stages are solved in an order which guarantees that all sub-problems (ie. problems associated with succeeding states) already have been solved and recorded (problems associated with goal states is initially known). Fig.3 illustrates one valid partitioning of the graph of fig. 1; problems in stage 1 are solved first, followed by problems in stage 2 , etc.

Fig. 3 also illustrates one of the main disadvantages of VI: Redundant problem solving. Problems (like those associated with node N2, N4, N6 and N7) which are not on a path leading from the start node will be solved, but they will never be reused. In contrast to RDP (where only problems on paths leading from the start node are represented and solved) there is in general no easy way (except processing the reduced state space prior to the optimization) to avoid this redundant problem solving in the VI algorithm.

The memory usage due to the recursive nature of the algorithm is the main disadvantage of RDP compared to VI. However, the memory complexity is linear in the depth of the problem or the number of stages and will therefore rarely cause any significant problems.

\subsection{Bounding}

Heuristic functions (which returns an under (over) estimate of the solution to a minimization (maximization) problem, see eg. Rich and Knight, 1991) might without effecting the solution to the problem reduce the problem space. The problem space is reduced by bounding subgraphs which with certainty will not lead to a better possible global solution than the current best known possible solution.

Kure (1995) defines a solution function for the basic model (RDPModel) which utilizes heuristic functions. The function is a special case of an algorithm known in AI as $A^{*}$ : The search strategy is a depth first search instead of an "intelligent" mixture of breadth first and depth first search as in $\mathrm{A}^{*}$.

A similar solution function might be defined for the general DP model and based on that function an $\mathrm{A}^{*}$ algorithm for stochastic problems might be derived.

\section{CONCLUSION}

Several aspects of DP have been examined and discussed in this paper. Some of these need special considerations.

The basic solution function, solution described in section 2 is seen to simply be a reformulation (with comb substituted by the infix operator ' + ' and the stage number interpreted as a state variable) of (1). Despite this very close and obvious relationship, the recursive approach towards DP presented in this paper, might seem new to most people dealing with DP and it appears difficult to find the approach described in literature (Kure, 1995).

The stepwise development of solution functions presented in this paper has shown that the use of memory functions is a distinct and excludable part of DP. In certain situations it might even appear more (time- and/or space- ) efficient to exclude a memory function from the solution function than to include it.

The recursive approach implies easy assessment of heuristic rules (and of decision space restricting rules in general). This feature might appear very beneficial in practical applications: Any appropriate rule is easily applied to the model and only states which according to 
the rules are on a path from the start state will be represented and solved. This easy application of heuristic rules is from an applicational point of view the most interesting feature. From a theoretical point of view the flexibility and generality of the models and solution functions is of greater interest and as indicated the approach might lead to a stochastic $\mathrm{A}^{*}$ algorithm and/or algorithms for solving problems that traditionally are represented as Bayesian Networks.

\section{REFERENCES}

Bellman, R. (1957): Dynamic Programming. Princeton University Press, Princeton, New Jersey.

Brassard, G. and P. Bratley (1988): Algorithms. Theory and Practice. Prentice-Hall International, Inc.

Jones, C. B. (1990): Systematic Software Development using VDM. Prentice Hall International (UK) Ltd.

Kure, H. (1995): Recursive Dynamic Programming. Specified and defined using VDM. Dina Notat No. 29. Dina, The Royal Veterinary and Agricultural University, Copenhagen.

Nemhauser, G. L. (1966): Dynamic Programming. John Wiley and Sons, Inc., NY.

Paulson, L. C. (1991): ML for the Working Programmer. Cambridge University Press, Cambridge

Shenoy, P. P. (1992): Valuation-based systems for Bayesian decision analysis, Operations Research 40, p 463-484.

Winston, P. H. (1992): Artificial Intelligence. AddisonWesley Publishing Company, NY.

\section{APPENDIX: A SML-IMPLEMENTATION}

Fragments of program:

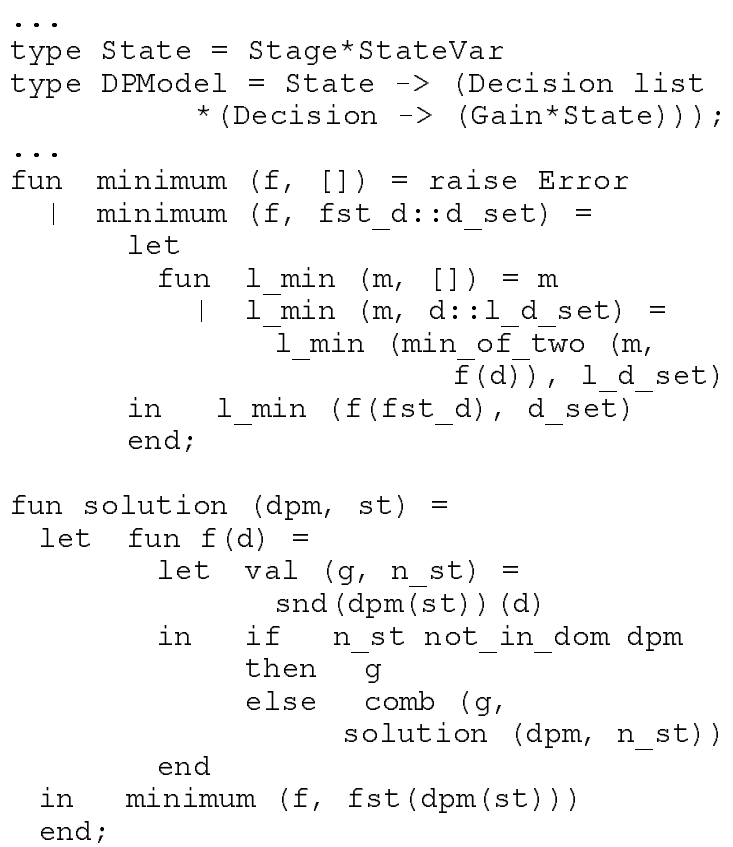

Corresponding fragments of output:

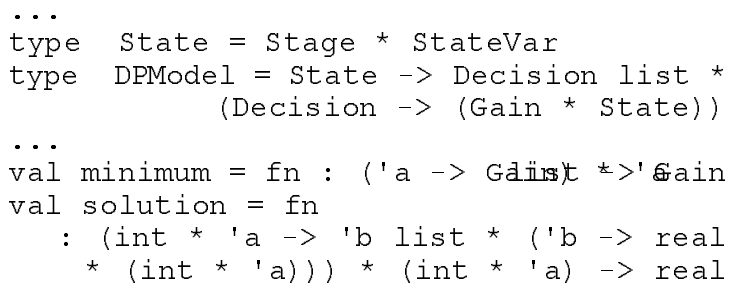

